\title{
Assessment of Photosynthetic Dysfunction in a Whole Tomato Plant with Chlorophyll Fluorescence Induction Imaging
}

\author{
Kotaro TAKAYAma ${ }^{1}$, Yoshiaki SAKaI ${ }^{1,2}$, Takako OIzUmi ${ }^{1}$ and Hiroshige Nishina ${ }^{1}$ \\ ${ }^{\prime}$ Faculty of Agriculture, Ehime University, 3-5-7 Tarumi, Matsuyama, Ehime 790-8566, Japan \\ ${ }^{2}$ ISEKI \& CO., LTD., 1 Yakura, Tobe, Iyo-gun, Ehime 791-2193, Japan
}

(Received November 22, 2010)

\begin{abstract}
Chlorophyll fluorescence is red light emitted from chlorophyll $a$ pigment and hence accurate measurement of this emission allows us to assess photosynthetic functions of the plant. The chlorophyll fluorescence induction imaging measures a chlorophyll fluorescence induction phenomenon. This phenomenon is a dynamic change in chlorophyll fluorescence intensity induced by illuminating plant body with an excitation light at a stable intensity under dark condition. The time course of the chlorophyll fluorescence intensity during this phenomenon is called induction curve. In this study, we developed a chlorophyll fluorescence induction imagining system for whole tomato plants as a first prototype for plant diagnosis in greenhouse. By using this system, we assessed the effects of sunlight exposure treatment, i.e. PPFD $1500 \mu \mathrm{mol} \mathrm{m}^{-2} \mathrm{~s}^{-1}$ for $2.5 \mathrm{~h}$, on the photosynthetic functions of a whole tomato plant of $1.1 \mathrm{~m}$ high. A substantial transformation of induction curve was observed between before and just after the treatment, but it was recovered in $6 \mathrm{~h}$ under dark condition. During the recovery process, the inflection points of $\mathrm{P}$ and $\mathrm{M}$, which are the characteristic inflections of an induction curve, showed different behaviour. The $\mathrm{M}$ kept lower values for $1.5 \mathrm{~h}$ after the sunlight exposure treatment even though the $\mathrm{P}$ had been almost recovered. This result suggests that the chlorophyll fluorescence induction imaging, especially concurrent monitoring of the images of $\mathrm{P}$ and $\mathrm{M}$ inflection points, is useful to detect invisible photosynthetic dysfunctions at whole plant level.
\end{abstract}

Keywords : diagnosis, induction curve, light stress, speaking plant approach

\section{INTRODUCTION}

As a most sophisticated strategy for environmental control in greenhouse, the concept of speaking plant approach (SPA) attracts a lot of attention. Originally, SPA defines that the optimal crop cultivation conditions should be based on the physiological status of the plants (Udink ten Cate et al., 1978; Hashimoto, 1980; 1989). The first and most important step of SPA is to obtain plant physiological information from a cultivated plant and then to judge whether or not the plant is healthy. Furthermore, the acquisition of the plant physiological information is preferable to be done without contact with the plant's body. For this purpose, imaging techniques have been intensively investigated.

Chlorophyll fluorescence imaging technique, originally developed by Omasa et al. (1987) and Daley et al. (1989), is a method to assess photosynthetic functions (Takayama et al., 2003; Omasa and Takayama, 2003; Takayama and Nishina, 2009). Chlorophyll fluorescence is a red light mis-

Corresponding author: Kotaro Takayama, fax : +81-89-946-9821, e-mail : takayama@agr.ehime-u.ac.jp 


\section{K. TAKAYAMA ET AL.}

sion from chlorophyll $a$ pigments and the intensity varies depending on the status of photosynthetic functions (Krause and Weis, 1991; Govindjee, 1995). Hence, the chlorophyll fluorescence imaging has been used to evaluate the heterogeneous distribution of photosynthetic activities over a leaf surface and has thus been applied to the detection of photosynthetic dysfunctions caused by biotic and abiotic stress factors (Daley, 1995; Genty and Meyer, 1995; Omasa and Takayama, 2002, 2003; Takayama et al., 2003).

There are two different methods, i.e. saturation pulse method and induction method, as the chlorophyll fluorescence imaging (Takayama and Nishina, 2009). Both the methods measure chlorophyll fluorescence emission, but the procedures of the measurement and analysis are completely different from each other. The induction method is preferable when one wants to conduct the chlorophyll fluorescence imaging in greenhouse, because a weak excitation light is enough to induce chlorophyll fluorescence emission for the measurement (Govindjee, 1995; Omasa and Takayama, 2002; Takayama et al., 2007; Takayama and Nishina, 2009; Takayama et al., 2009). Although no widely used parameters are derived from the induction method, it has a great potential as a method for plant health monitoring of whole plants and plant canopies.

In our previous study, we reported that the photosynthetic dysfunctions in single tomato leaves, which was caused by artificial strong light irradiation treatment, was detectable with chlorophyll fluorescence induction imaging (Takayama et al., 2009). In this study, we extended the chlorophyll fluorescence induction imaging technique at a single leaf level to whole plant level and developed a chlorophyll fluorescence imagining system for whole tomato plants. By using this system, we evaluated the effect of sunlight exposure treatment on the photosynthetic functions of a whole tomato plant.

\section{MATERIALS AND METHODS}

\section{Chlorophyll fluorescence induction phenomenon}

A part of the absorbed light energy unused for photosynthetic reaction is emitted as red light, i.e. chlorophyll fluorescence. An accurate measurement of this emission allows us to detect invisible photosynthetic dysfunctions. Imaging of chlorophyll fluorescence induction phenomenon is a useful technique to evaluate the status of photosynthetic functions of in-situ plant under greenhouse condition.

The chlorophyll fluorescence induction phenomenon, a dynamic change in chlorophyll fluorescence intensity, is induced by illuminating a dark-adapted green leaf with a stable intensity excitation light (Govindjee, 1995; Omasa and Takayama, 2002). And the time course of the chlorophyll fluorescence intensity during the induction phenomenon, which is plotted along a logarithmic time axis, is called 'Induction curve' (Takayama and Nishina, 2009). The shape of the induction curve varies depending on the status of photosynthetic functions of the plant (Govindjee, 1995; Omasa and Takayama, 2002; Takayama et al., 2009).

Figure 1 shows a schematic of a typical chlorophyll fluorescence induction curve. The typical curve has characteristic inflection points (I-D-P-S-M-T) (Govindjee, 1995; Omasa and Takayama, 2002; Takayama and Nishina, 2009). The fast phase (from I to P) takes a few seconds after the start of excitation. In general, the fast phase is closely related with the redox reactions of $\mathrm{Q}_{\mathrm{A}}$, the primary electron acceptor of photosystem II (PS II) (Krause and Weis, 1991). In contrast, the decrease in chlorophyll fluorescence intensity observed between points $\mathrm{P}$ and $\mathrm{T}$ requires a couple of minute. The slow phase varies depending on various reactions such as photosynthetic photochemical reaction in thylakoid membrane, heat dissipation processes, and carbon fixation in stroma (Krause and Weis, 1991).

Chlorophyll fluorescence induction imaging system for whole tomato plant

Figure 2 shows a schematic diagram of the developed chlorophyll fluorescence induction 


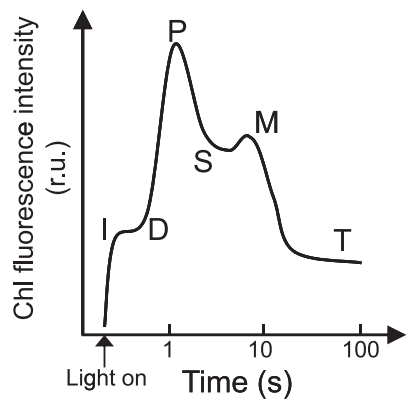

Fig. 1 Schematic of a typical chlorophyll fluorescence induction curve induced by an excitation light. The "Light on" indicates the starting point of the excitation light irradiation. The characters along the curve (I, D, P, S, M and T) indicate the representative inflection points of the induction curve.
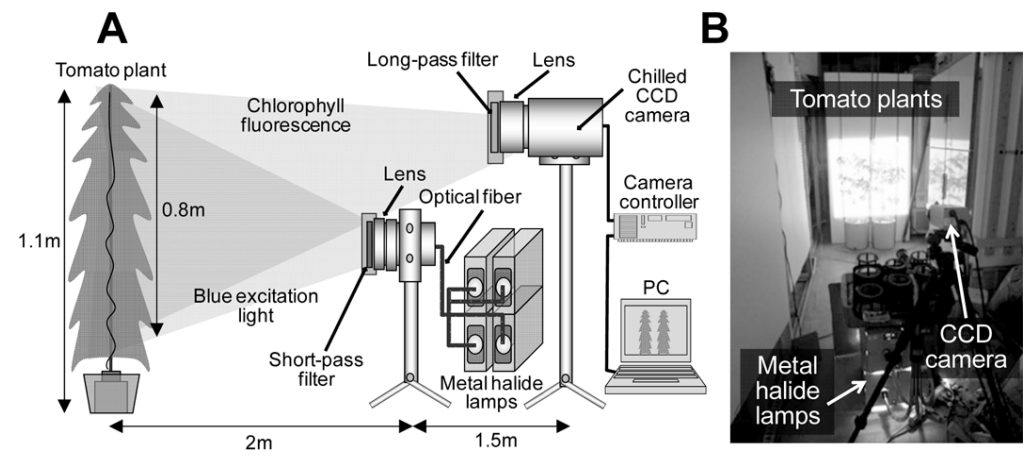

Fig. 2 (A) Chlorophyll fluorescence imaging system for whole tomato plants. The intensity of the blue excitation light at the plant surface was about PPFD $10 \mu \mathrm{mol} \mathrm{m}^{-2} \mathrm{~s}^{-1}$. (B) A picture of chlorophyll fluorescence imaging of two whole tomato plants under dark condition in a laboratory.

imaging system for whole tomato plant (Fig. 2-A) and a picture of the chlorophyll fluorescence imaging of two whole tomato plants under dark condition in a laboratory (Fig. 2-B). An optical fiber filtered with a short-pass filter $(\lambda<620 \mathrm{~nm}$, Corning 4-96) was used to condense light from four $180 \mathrm{~W}$ metal halide lamps (LS-M180, Sumita Optical Glass, Inc.) into a blue excitation light. The blue excitation light illuminates a $0.8 \mathrm{~m} \times 0.8 \mathrm{~m}$ area, which covers all the leaves of two $1.1 \mathrm{~m}$ high tomato plants located at a distance of $2 \mathrm{~m}$ from the light source. The intensity of the blue excitation light was about PPFD $10 \mu \mathrm{mol} \mathrm{m}^{-2} \mathrm{~s}^{-1}$ on the plant surface. Application of the blue excitation light for $100 \mathrm{~s}$ induces the chlorophyll fluorescence induction phenomenon. The induced chlorophyll fluorescence was captured with a CCD camera (C5985-02, Hamamatsu Photonics K.K.) filtered with a long-pass filter $(\lambda>680 \mathrm{~nm}$, Corning 2-64), which was located at a distance of $3.5 \mathrm{~m}$ from the plants. By using the camera controller, the shutter speed and gain of the CCD camera were set at $0.03 \mathrm{~s}$ and +8 , respectively. The time resolution was $30 \mathrm{fps}$ and the image resolution was 1024 (W) $\times 768(\mathrm{H})$ pixels. All the images were recorded on the hard disk of a laptop computer (VAIO VGN-FS20 typeF, Sony Corporation). After the image capturing, the captured images were converted into BMP format images and then the images were analyzed with a self developed Visual Basic 6.0 software, i.e. extraction of plant area, calculation of average chlorophyll fluorescence intensity of each BMP image.

\section{Plant material}

Tomato (Solanum lycopersicum L., Momotaro 8) seeds were sown in spongy material on 2nd June 2007 and transplanted to rock wool cubes $(10 \mathrm{~cm} \mathrm{[W] \times 10} \mathrm{cm} \mathrm{[H]} \times 10 \mathrm{~cm}[\mathrm{D}])$ on 27 th July 


\section{K. TAKAYAMA ET AL.}

2007 after germination, and then grown for 8 weeks in an experimental greenhouse located in Faculty of Agriculture, Ehime University. No electrical active air conditioning instruments but ventilation windows on the roof and sidewall were equipped in the greenhouse. Average air temperature and relative humidity in the greenhouse were $26.5^{\circ} \mathrm{C}$ and $46 \%$ during day and $19.0^{\circ} \mathrm{C}$ and $63 \%$ at night. The plants were watered daily with a nutrient solution (A-type recipe of Otsuka House Solution, Otsuka Chemical Co., Ltd.). The height of the tomato plants was about $1.1 \mathrm{~m}$ and the height of the leaf layer was about $0.8 \mathrm{~m}$ when the plants were used for the experiment.

Experimental procedure

Two tomato plants were used for the experiment. The plants were moved into a laboratory from the greenhouse and adapted to the dark condition for $12 \mathrm{~h}$ (Fig. 3-A). After the dark adaptation, an initial chlorophyll fluorescence induction imaging was done. Figure 3-B shows a chlorophyll fluorescence intensity image of the two tomato plants captured with the chlorophyll fluorescence induction imaging system (Fig. 2). Then, the right plant in Fig. 3 was transferred outside and exposed to sunlight for $2.5 \mathrm{~h}$ (Light-treated). The average intensity of sunlight during the treatment was approximately PPFD $1500 \mu \mathrm{mol} \mathrm{m}^{-2} \mathrm{~s}^{-1}$ at the top of the plant. After the sunlight exposure treatment, the plant was immediately transferred into the dark laboratory and put in the original position. During the sunlight exposure treatment, another plant, the left plant in Fig. 3, was continuously kept in the dark condition (Control). The chlorophyll fluorescence induction imaging was done just after and at $0.5,1.5$ and $6 \mathrm{~h}$ after the sunlight exposure treatment. All the chlorophyll fluorescence imaging was conducted under dark condition.

\section{RESULTS AND DISCUSSION}

Figure 4 shows changes in the visual appearance of the Light-treated tomato plant before and during $6 \mathrm{~h}$ after the sunlight exposure treatment. The images of Fig. 4 were the intensity-corrected chlorophyll fluorescence images for the observation of the appearance change. A slight wilting was observed just after the treatment and it remained for $0.5 \mathrm{~h}$ after the treatment (White arrows in Fig. 4). But the wilting was almost disappeared at $1.5 \mathrm{~h}$ after the treatment. The slight wilting observed in the Light-treated plant just after the sunlight exposure treatment might be caused by water stress, which was induced by transferring the plant from the dark laboratory condition to full sunlight condition. The extent of the wilting was very slight and such level of wilting was sometimes observed in the greenhouse, hence the direct effect of the wilting on the photosynthesis seems not critical in this study.

Figure 5 shows changes in the chlorophyll fluorescence induction curves of the tomato plants before and during $6 \mathrm{~h}$ after the sunlight exposure treatment. Each value of chlorophyll fluorescence intensity was a mean value of all the pixel values within the plant area in each chlorophyll fluores-

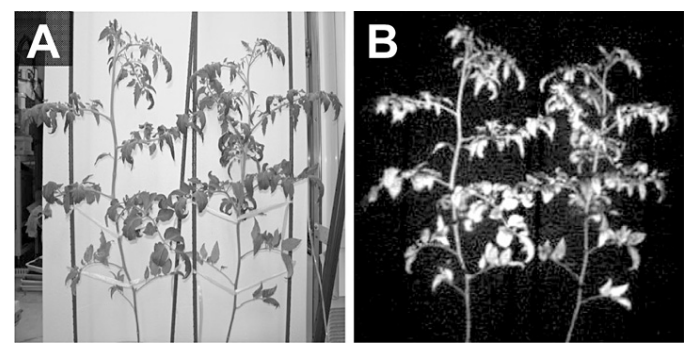

Fig. 3 ( A) A visual image of the two whole tomato plants used for the experiment. The left plant was continuously placed under dark condition (Control) and the right one was exposed to sunlight (PPFD 1500 $\mu \mathrm{mol} \mathrm{m} \mathrm{m}^{-2} \mathrm{~s}^{-1}$ ) for $2.5 \mathrm{~h}$ (Light-treated). (B) A chlorophyll fluorescence image captured with the chlorophyll fluorescence induction imaging system shown in Fig. 2. 

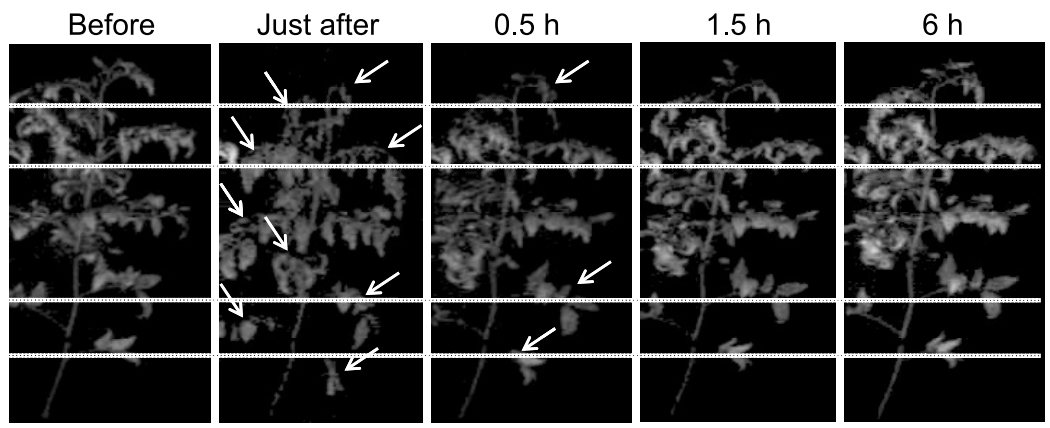

Fig. 4 Changes in the visual appearance of the Light-treated plant before and during $6 \mathrm{~h}$ after the sunlight exposure treatment. These images are the intensity-corrected chlorophyll fluorescence images for the observation of the appearance change. The white arrows indicate the wilting parts and the dotted horizontal lines represent the same levels.

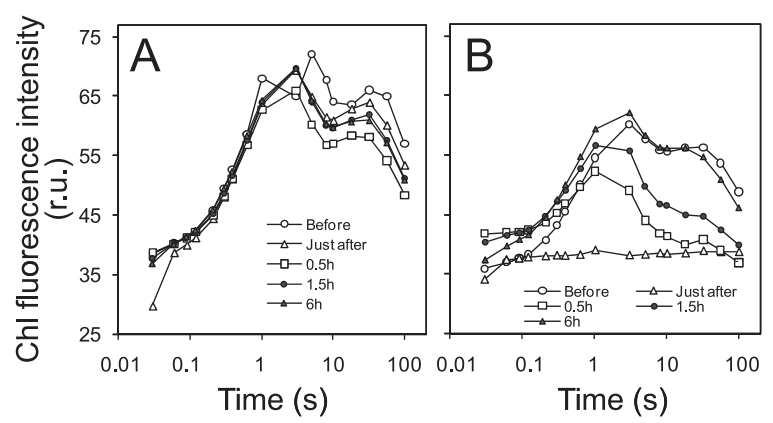

Fig. 5 Changes in chlorophyll fluorescence induction curves of Control (A) and Light-treated (B) before and during $6 \mathrm{~h}$ after the sunlight exposure treatment.

cence image. The Control plant showed typical shapes of induction curve and maintained a similar form throughout the experiment (Fig. 5-A). The P, S and M inflections of the Control plant were observed at 2-3, 8-10 and about $30 \mathrm{~s}$ after the commencement of the excitation light irradiation, respectively. And the chlorophyll fluorescence intensities at $\mathrm{P}$ and $\mathrm{M}$ inflections were almost stable and the values were ranging from 66 to 72 for $\mathrm{P}$ and from 58 to 66 for $\mathrm{M}$ throughout the experiment. This result suggested that the developed chlorophyll fluorescence induction imagining system is able to obtain similar chlorophyll fluorescence induction curves from a same plant in a similar physiological status.

The induction curve of the Light-treated plant measured before the sunlight exposure treatment showed a typical shape of chlorophyll fluorescence induction curve and the chlorophyll fluorescence intensities at the $\mathrm{P}, \mathrm{M}$ and $\mathrm{S}$ inflections were 60, 56 and 56, respectively (Fig. 5-B). Before the treatment, the chlorophyll fluorescence intensity at the inflection point of $\mathrm{P}$ of the Control plant was slightly higher compared with that of the Light-treatment plant and the shapes of the two induction curves were not completely same ( $\bigcirc$ in Fig. 5-A and B). These differences in the induction curves were mainly due to the individual differences in total leaf area and leaf angle of the two plants. Such differences in chlorophyll fluorescence induction curves were also observed at the level of individual leaves (Takayama et al., 2009).

However, the induction curve of the Light-treated plant was substantially flattened just after the sunlight exposure treatment, and a small $\mathrm{P}$ inflection was recognized at $1 \mathrm{~s}$ after the commencement of the excitation light irradiation but $\mathrm{S}$ and $\mathrm{M}$ inflections were not identified in the induction 


\section{K. TAKAYAMA ET AL.}

curve ( $\triangle$ in Fig. 5-B). This flattening of induction curve caused by the sunlight exposure treatment would be attributed to the activation of heat dissipation process that dissipates absorbed excessive light energy as heat (Krause and Weis, 1991). Similar phenomenon was observed in our previous study with individual leaves (Takayama et al., 2009). In spite of the significant transformation of the induction curve, the shape of the induction curve had started to recover at $0.5 \mathrm{~h}$ after the treatment ( $\square$ in Fig. 5-B) and apparent P, S and M inflections were observed at $1.5 \mathrm{~h}$ after the treatment ( in Fig. 5-B). Furthermore, the induction curve measured at $6 \mathrm{~h}$ after the treatment showed almost similar shape of that measured before the treatment ( $\boldsymbol{\Delta}$ in Fig. 5-B). This rapid recovery might be due to the prompt relaxation of heat dissipation process under dark condition (Krause and Weis, 1991; Müller et al., 2001; Takayama et al., 2009).

Figure 6 shows changes in chlorophyll fluorescence intensity images at the inflection point of $\mathrm{P}$, i.e. $\mathrm{P}$ images, before and during $6 \mathrm{~h}$ after the sunlight exposure treatment. The $\mathrm{P}$ images of the Control plant were almost similar throughout the experiment. On the other hand, a significant decrease in chlorophyll fluorescence intensity of the Light-treated plant was observed just after the treatment. After the decrease, the chlorophyll fluorescence intensity of the Light-treated plant was immediately recovered at $0.5 \mathrm{~h}$ after the treatment. Figure 7 shows changes in chlorophyll fluorescence intensity images at the inflection point of $\mathrm{M}$, i.e. $\mathrm{M}$ images, before and during $6 \mathrm{~h}$ after the sunlight exposure treatment. The $\mathrm{M}$ images of the Control plant were almost similar throughout the experiment as well as the P images. For the Light-treated plant, a significant decrease in chlorophyll fluorescence intensity was observed just after the treatment and it continued for at least 1.5 $\mathrm{h}$ after the treatment. After that, the chlorophyll fluorescence intensity was completely recovered at $6 \mathrm{~h}$ after the treatment.

Figure 8 shows changes in relative chlorophyll fluorescence intensities at the inflection points of $\mathrm{P}$ and $\mathrm{M}$ during $6 \mathrm{~h}$ after the sunlight exposure treatment. The relative chlorophyll fluorescence intensity is the ratio of the chlorophyll fluorescence intensity at each measurement time point to the initial intensity measured before the treatment. For the Control plant, both the relative chlorophyll fluorescence intensities of $\mathrm{P}$ ( $\bigcirc$ in Fig. 8) and M ( $\square$ in Fig. 8) kept at almost constant value ranging from 0.89 to 1.02 throughout the experiment. For the Light-treated plant, the relative chlorophyll fluorescence intensity of $\mathrm{P}(\mathrm{in}$ Fig. 8 ) decreased to 0.64 , i.e. $36 \%$ decrease compared with the initial intensity, just after the treatment but immediately increased to 0.87 at $0.5 \mathrm{~h}, 0.94$ at 1.5

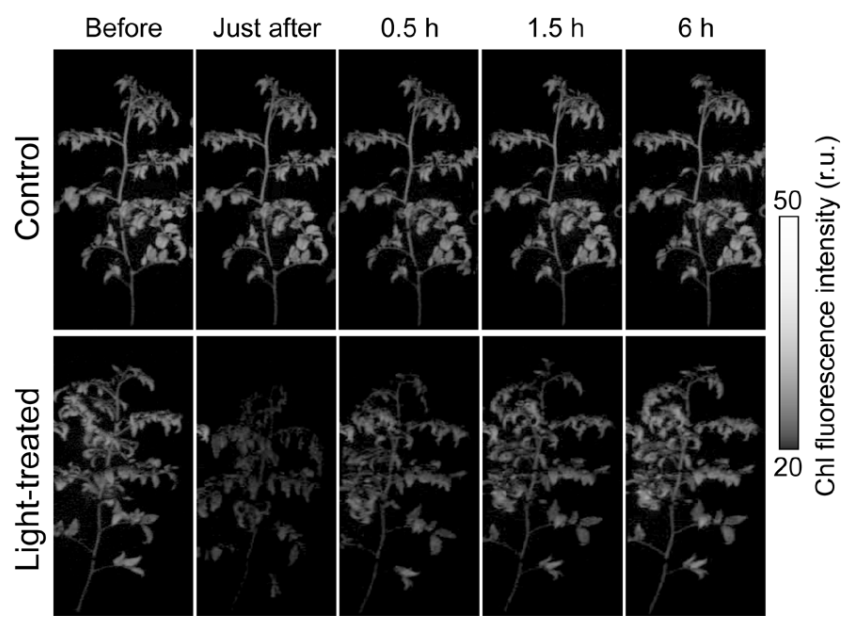

Fig. 6 Changes in chlorophyll fluorescence intensity images at the inflection point of P, i.e. maximum peak of a chlorophyll fluorescence induction curve, before and during $6 \mathrm{~h}$ after the sunlight exposure treatment. 


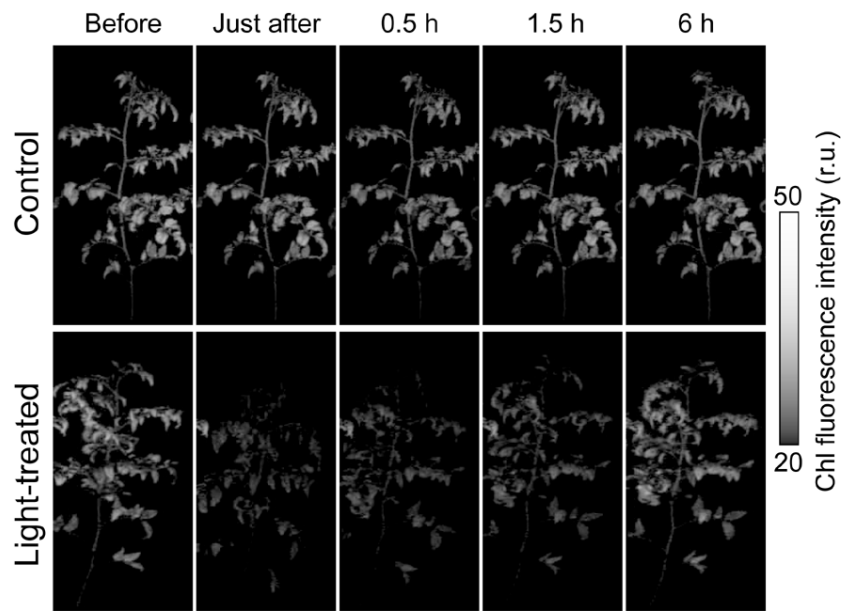

Fig. 7 Changes in chlorophyll fluorescence intensity images at the inflection point of $\mathrm{M}$, i.e. secondary peak of a chlorophyll fluorescence induction curve, before and during $6 \mathrm{~h}$ after the sunlight exposure treatment.

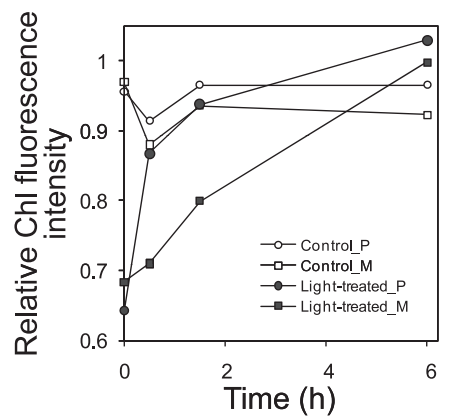

Fig. 8 Changes in relative chlorophyll fluorescence intensities at $\mathrm{P}$ and $\mathrm{M}$ inflection points of the Control and Light-treated plants during $6 \mathrm{~h}$ after the sunlight exposure treatment. The values is the ratio of the chlorophyll fluorescence intensity at each measurement time point to the initial intensity measured before the treatment.

$\mathrm{h}$, and 1.03 at $6 \mathrm{~h}$ after the treatment. This result suggested that the heat dissipation process caused by the sunlight exposure treatment was mainly consisted of the component related to a xanthophyll cycle because its relaxation is rapid and generally completes within $1 \mathrm{~h}$ after the commencement of relaxation under dark condition (Krause and Weis, 1991; Müller et al., 2001).

On the other hand, the relative chlorophyll fluorescence intensity of M ( $\square$ in Fig. 8) decreased to 0.68 just after the sunlight exposure treatment, and kept relatively lower values for $1.5 \mathrm{~h}$ after the treatment, i.e. 0.71 at $0.5 \mathrm{~h}$ and 0.79 at $1.5 \mathrm{~h}$ after the treatment. Then, it recovered to 0.99 at $6 \mathrm{~h}$ after the treatment. This recovery process of the inflection point of $\mathrm{M}$ was not similar to that of the inflection point of $\mathrm{P}(\mathrm{in}$ Fig. 8). The value of $\mathrm{M}$ was still low at $1.5 \mathrm{~h}$ after the treatment ( in Fig. 5-B, Fig. 7, $\square$ in Fig. 8), even though the depression of $P$ and wilting observed just after the treatment had been almost recovered (Fig. 4, in Fig. 5-B, Fig. 6, $\bigcirc$ in Fig. 8). This suggested that the recovery of the inflection point of $\mathrm{M}$ from the depression caused by the sunlight exposure treatment took longer time compared with the recovery of the inflection point of P. Similar phenomenon was observed in our previous study with individual leaves (Takayama et al., 2009). The reason of this phenomenon is not clear, but it seems that the recovery of the inflection point 


\section{K. TAKAYAMA ET AL.}

of M might be governed by a potential activation ability of heat dissipation process, not by the heat dissipation process itself, because the transition between the inflection points of $\mathrm{P}$ and $\mathrm{S}-\mathrm{M}$ of an induction curve is mainly induced by the activation of heat dissipation process, i.e. activation of xanthophyll synthesis via a xanthophyll cycle (Krause and Weis, 1991; Müller et al., 2001).

\section{CONCLUSION}

We developed a chlorophyll fluorescence induction imagining system for whole tomato plants, which measures chlorophyll fluorescence induction phenomenon induced by a weak blue excitation light. By using this system, we evaluated the effect of sunlight exposure treatment on the photosynthetic function of a whole tomato plant. A substantial activation of heat dissipation process after the treatment was successfully detected by imaging at $\mathrm{P}$ and $\mathrm{M}$ inflection points of the induction curve. The relaxation of the activated heat dissipation process under dark condition was also quantitatively evaluated. Furthermore, the chlorophyll fluorescence images at the inflection point of $\mathrm{M}$ revealed that the potential effect caused by the sunlight exposure treatment was still remained in the Light-treated plant for $1.5 \mathrm{~h}$ after the treatment even though the depression of the inflection point of $\mathrm{P}$ and wilting observed just after the treatment had been almost recovered. These results suggested that the continuous monitoring of both the inflection points of $\mathrm{P}$ and $\mathrm{M}$ with the chlorophyll fluorescence induction imagining system is useful to detect photosynthetic dysfunctions in whole plant.

\section{REFERENCES}

Daley, P. F., Raschke, K., Ball, J. T., Berry, J. A. 1989. Topography of photosynthetic activity of leaves obtained from video images of chlorophyll fluorescence. Plant Physiol. 90: 1233-1238.

Daley, P. F. 1995. Chlorophyll fluorescence analysis and imaging in plant stress and disease. Can. J. Plant Pathol. 17: 167-173.

Genty, B., Meyer, S. 1995. Quantitative mapping of leaf photosynthesis using chlorophyll fluorescence imaging. Aust. J. Plant Physiol. 22: 277-284.

Govindjee. 1995. Sixty-three years since Kautsky: Chlorophyll a fluorescence. Aust. J. Plant Physiol. 22: 131-160.

Hashimoto, Y. 1980. Computer control of short term plant growth by monitoring leaf temperature. Acta Hort. 106: 139-146.

Hashimoto, Y. 1989. Recent strategies of optimal growth regulation by the speaking plant concept. Acta Hort. 260: 115-121.

Krause, G. H., Weis, E. 1991. Chlorophyll fluorescence and photosynthesis: the basics. Annu. Rev. Plant Physiol. Plant Mol. Biol. 42: 313-349.

Müller, P., Li, X.P., Niyogi, K. K. 2001. Non-photochemical quenching: a response to excess light energy. Plant Physiol. 125: $1558^{-1566 . ~}$

Omasa, K., Shimazaki, K., Aiga, I., Larcher, W., Onoe, M. 1987. Image analysis of chlorophyll fluorescence transients for diagnosing the photosynthetic system of attached leaves. Plant Physiol. 84: 748-752.

Omasa, K., Takayama, K. 2002. Image instrumentation of chlorophyll a fluorescence for diagnosis photosynthetic injury. In "Air Pollution and Plant Biotechnology" (ed. by Omasa, K., Saji, H., Youssefian, S., Kondo, N.), Springer-Verlag, Tokyo, p 287-308.

Omasa, K., Takayama, K. 2003. Simultaneous measurement of stomatal conductance, non-photochemical quenching, and photochemical yield of photosystem II in intact leaves by thermal and chlorophyll fluorescence imaging. Plant Cell Physiol. 44: 1290-1300.

Takayama, K., Konishi, A., Omasa, K. 2003. Diagnosis of invisible photosynthetic injury caused by a herbicide (Basta) with chlorophyll fluorescence imaging system. Agricultural Engineering International: the CIGR Journal of Scientific Research and Development, Manuscript IT 03001 5: 1-12.

Takayama, K., Nishina, H., Sakai, K. 2007. Analysis of photosynthetic functions of Hypnum plumaeforme using chlorophyll fluorescence imaging technique. Eco-Engineering 19: 49-57. 


\section{FLUORESCENCE IMAGING OF PLANT}

Takayama, K., Nishina, H. 2009. Chlorophyll fluorescence imaging of the chlorophyll fluorescence induction phenomenon for plant health monitoring. Environ. Control Biol. 47: 101-109.

Takayama, K., Nishina, H., Oizumi, T., Arima, S., Hatou, K. 2009. Detection of photoinhibition in tomato leaves with imaging of chlorophyll fluorescence induction phenomenon induced by weak actinic light. EcoEngineering 21: 69-74.

Udink ten Cate, A. J., Bot, G. P. A., van Dixhoorn, J. J. 1978. Computer control of greenhouse climates. Acta Hort. 87: 265-272. 